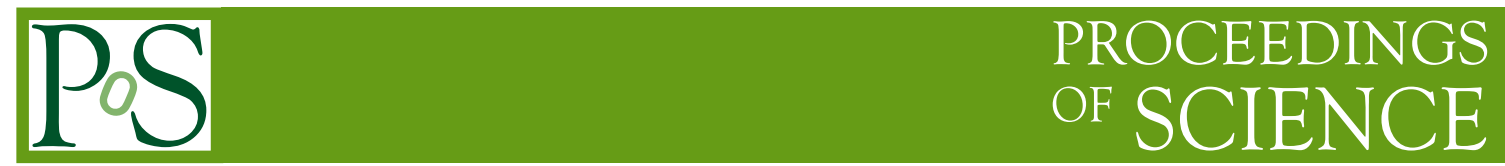

\title{
Status of Higgs Boson Couplings and Searches
}

\section{Peter Onyisi*, on behalf of the ATLAS and CMS Collaborations}

University of Texas at Austin, Austin, TX 78712, United States

Email: ponyisi@utexas.edu

The status of Higgs sector studies at the Large Hadron Collider is reviewed. The latest extractions of couplings and properties of the $125 \mathrm{GeV}$ Higgs boson from the Run 1 data of the ATLAS and CMS experiments are discussed. Searches for additional Higgs boson states beyond that of the Standard Model are presented.

Flavor Physics \& CP Violation 2015

May 25-29, 2015

Nagoya, Japan

${ }^{*}$ Speaker. 


\section{Introduction}

After the discovery of a $125 \mathrm{GeV}$ Higgs boson by the ATLAS [1] and CMS [2] Collaborations, effort has focused on studying its properties and searching for potential additional particles in the electroweak symmetry breaking sector. There are many complementarities between direct study of the Higgs boson and the lower-energy probes typical of flavor physics. Due to its role in providing masses to fermions, the Higgs boson generates the CKM matrix, and can potentially provide a source of flavor-changing neutral currents. An extended Higgs sector, or mixing of the Higgs boson with other particles, may generate additional sources of $C P$-violation. Direct and indirect searches for a charged scalar $\mathrm{H}^{+}$cover similar regions of parameter space. Additional light particles may be searched for both in low- and high-energy experiments.

These proceedings cover recent Higgs physics results from the LHC experiments with particular relevance to flavor physics. Searches for additional heavy Higgs sector states other than $\mathrm{H}^{+}$ will only be briefly discussed.

\section{Couplings and Properties of the $125 \mathrm{GeV}$ Higgs Boson}

\subsection{Mass}

The fully-reconstructible Higgs boson decay modes $H \rightarrow \gamma \gamma$ and $H \rightarrow Z Z^{*} \rightarrow 4 \ell$ have been used to measure the Higgs boson mass with high precision at both ATLAS and CMS. The ATLAS and CMS Collaborations have combined their measurements in these two channels to obtain a value

$$
m_{H}=125.09 \pm 0.21 \text { (stat) } \pm 0.11 \text { (syst) GeV }
$$

a $0.2 \%$ measurement [3]. The largest systematic uncertainties are related to electromagnetic calorimeter energy response for photons and electrons in the two detectors and to the muon momentum measurement in CMS.

\subsection{Couplings}

\subsubsection{Formalism}

Both ATLAS [4] and CMS [5] have measured the couplings of the Higgs boson using the so-called $\kappa$-formalism [6]. In this parametrization, the strength of the Higgs boson coupling to the Standard Model (SM) particle $X$ is scaled by $\kappa_{X}$. In cases where the Higgs boson can decay to $X$, the partial width obeys $\Gamma_{X}=\kappa_{X}^{2} \Gamma_{X, S M}$. Effects such as higher dimension operators with different Lorentz structures are ignored in this approximation.

One can add a branching fraction $\mathrm{BR}_{i, u}$ of the Higgs boson to invisible particles (e.g. dark matter) or to undetected final states (anomalous decays that have not been searched for, but with visible final states). The distinction is relevant when explicit searches for invisible Higgs boson decays are combined with indirect constraints based on visible decays. With on-shell measurements only, it is possible to compensate for a common scaling of the SM couplings $\kappa_{X}=\kappa, \kappa>1$, by adding invisible/BSM decays $\mathrm{BR}_{i, u}=1-1 / \kappa^{2}$.

The data provide strong constraints on the gluon-gluon fusion production rate (through $g g \rightarrow$ $H \rightarrow W W^{*}, \gamma \gamma, Z Z^{*}$ ) and the $H \rightarrow \gamma \gamma$ rate (through $g g \rightarrow H \rightarrow \gamma \gamma$ ). Both the $g g H$ and $\gamma \gamma H$ couplings 
arise at loop level in the SM. It is possible to assume that no new physics contributes to these loops and that any modification in these rates arises purely from modifications to tree-level Higgs couplings; this is termed resolving the loop. For example, the expression for the modification to the effective $H \rightarrow \gamma \gamma$ coupling, $\kappa_{\gamma}$, can be expressed as

$$
\kappa_{\gamma}=1.59 \kappa_{W}^{2}+0.07 \kappa_{t}^{2}-0.66 \kappa_{W} \kappa_{t}
$$

reflecting the significant destructive interference between $W$ and top quark loops in the decay. This parametrization provides very strong constraints on $\kappa_{t}$ (also from the $g g H$ vertex, which is completely top quark-loop dominated), and is also sensitive to the relative signs of $\kappa_{W}$ and $\kappa_{t}$, at the expense of losing any sensitivity to new physics that cannot be parametrized as modifications to $\kappa_{t}$ and $\kappa_{W}$.

Alternatively, $\kappa_{\gamma}$ and $\kappa_{g}$ can be allowed to be modified independent of $\kappa_{t}$ and $\kappa_{W}$; in this case the loops are unresolved. Because the model now has additional parameters additional information is needed to fully constrain the parameters. In particular this requires measurements of processes like $t \bar{t} H$ production, which has not yet been observed.

\subsubsection{On-shell inputs to the Coupling Measurement}

ATLAS and CMS input their suite of Standard Model Higgs boson measurements and searches to their coupling combinations in grand fits to data. These are summarized in Fig. 1. In general the results are reported in terms of signal strength parameters $\mu$, which express the ratio of observed signal to SM expectation, including theoretical uncertainties in their errors.
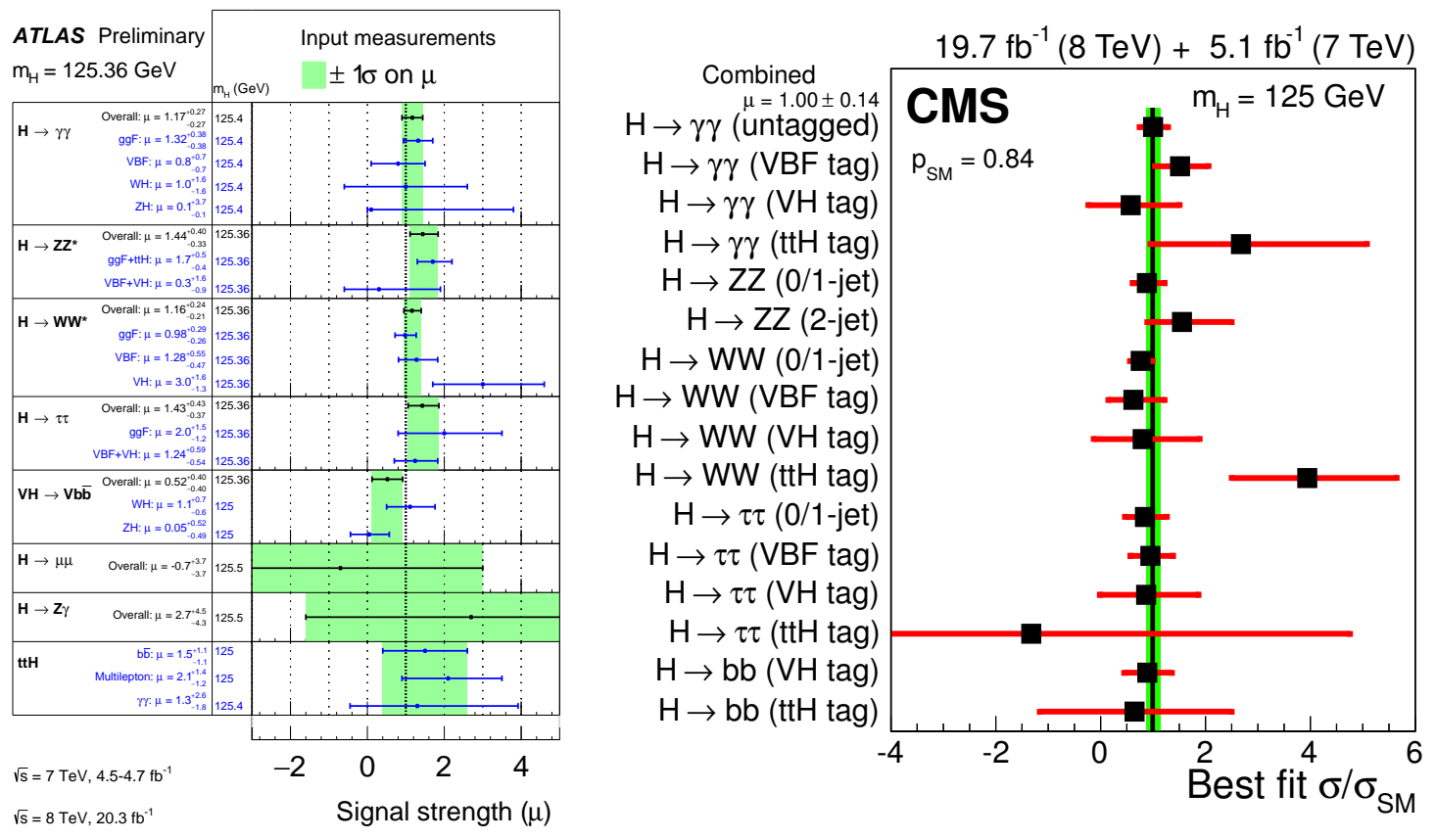

Figure 1: Inputs to the ATLAS [4] (left) and CMS [5] (right) Higgs coupling extraction from on-shell measurements. 


\subsubsection{Off-shell inputs to the Coupling Measurement}

The lineshape of the Higgs boson at $s \gg m_{H}^{2}$ is complex due to threshold effects; once the mass exceeds $2 m_{V}(V=W, Z)$, the Higgs boson can decay to on-shell pairs of $W$ or $Z$ bosons and the partial width to those final states is greatly enhanced. The off-shell production rate is also sensitive to passing the threshold $\sqrt{s}>2 m_{t}$, at which point the top quark loop in $g g \rightarrow H$ can no longer be treated in the infinite top mass approximation. Looking at a Breit-Wigner expression for the scattering cross section,

$$
\sigma(i \rightarrow H \rightarrow f) \propto \frac{g_{i}^{2} g_{f}^{2}}{\left(s-m_{H}^{2}\right)^{2}+m_{H}^{2} \Gamma_{H}^{2}},
$$

one observes that the $m_{H}^{2} \Gamma_{H}^{2}$ term is important when $s \sim m_{H}^{2}$, while when $s \gg m_{H}^{2}$ the width is not relevant. A measurement of the line shape far off-shell can therefore constrain the width and the enhancement of the $H \rightarrow Z Z / W W$ rate above $2 m_{V}$ provides a mechanism to access the Higgs boson lineshape at high $s$.

In practice the cross section for $g g \rightarrow H \rightarrow V V$ in the SM is smaller than that for direct $g g \rightarrow V V$, and interference between the two is in fact destructive. It is not currently possible to sufficiently constrain the direct term theoretically to permit a measurement of the SM-level interference between direct and Higgs-mediated processes. However if the Higgs process occurred at significantly higher rates than the SM, the Higgs term would dominate and produce a measurable excess. ATLAS [7] and CMS [8] have used high mass $Z Z$ and $W W$ production measurements and the assumption that the couplings are $s$-independent to constrain the Higgs boson width:

$$
\begin{aligned}
\Gamma_{H} / \Gamma_{H, S M} & <4.5-7.5(6.5-11.2 \text { expected })(\mathrm{ATLAS}) @ 95 \% \mathrm{CL} \\
& <5.4(8.0 \text { expected })(\mathrm{CMS}) @ 95 \% \mathrm{CL}
\end{aligned}
$$

where the range in the ATLAS results arises from different assumptions on the NLO corrections to $g g \rightarrow V V$ (CMS assumes these corrections are the same for signal and background). These limits are two orders of magnitude stronger than those from direct measurement, which is limited by detector resolution.

\subsubsection{Results}

Both ATLAS and CMS see evidence for Higgs boson production in the gluon-gluon fusion mechanism in individual channels at significance exceeding $5 \sigma$, and this mode is well established. The expected and observed significance of the signals for the other production modes is shown in Table 1. In both experiments the measured $t \bar{t} H$ rate is high, leading to a more significant signal than expected.

If no BSM particles or new Higgs boson decay modes are allowed, fairly strong constraints can be obtained for the couplings to $W, Z$, top quark, and $\tau$, with uncertainties of order $\lesssim 20 \%$ in each experiment (and 14\% in the case of the $W$ coupling). The results are shown in Fig. 2. The bottom quark coupling is less well established (order 30-35\% uncertainty in the coupling) and for muons a limit can be set, as there is no evidence of this mode yet. The couplings are consistent with the SM at $\approx 1 \sigma$ or better for all couplings.

A more generic model (using only on-shell measurements) can be achieved by dropping assumptions on the Higgs boson width. Because it is possible to scale all couplings by a common 
Table 1: Significance of Higgs boson production modes other than gluon-gluon fusion. Values given are observed and (expected) significance.

\begin{tabular}{lcc}
\hline \hline Mechanism & ATLAS & CMS \\
\hline Vector boson fusion (VBF) & $4.3 \sigma(3.8 \sigma)$ & $3.7 \sigma(3.3 \sigma)$ \\
$W, Z$ associated production $(V H)$ & $2.6 \sigma(3.1 \sigma)$ & $2.7 \sigma(2.9 \sigma)$ \\
$t \bar{t}$ associated production $(t \bar{t} H)$ & $2.4 \sigma(1.5 \sigma)$ & $3.5 \sigma(1.2 \sigma)$ \\
\hline \hline
\end{tabular}
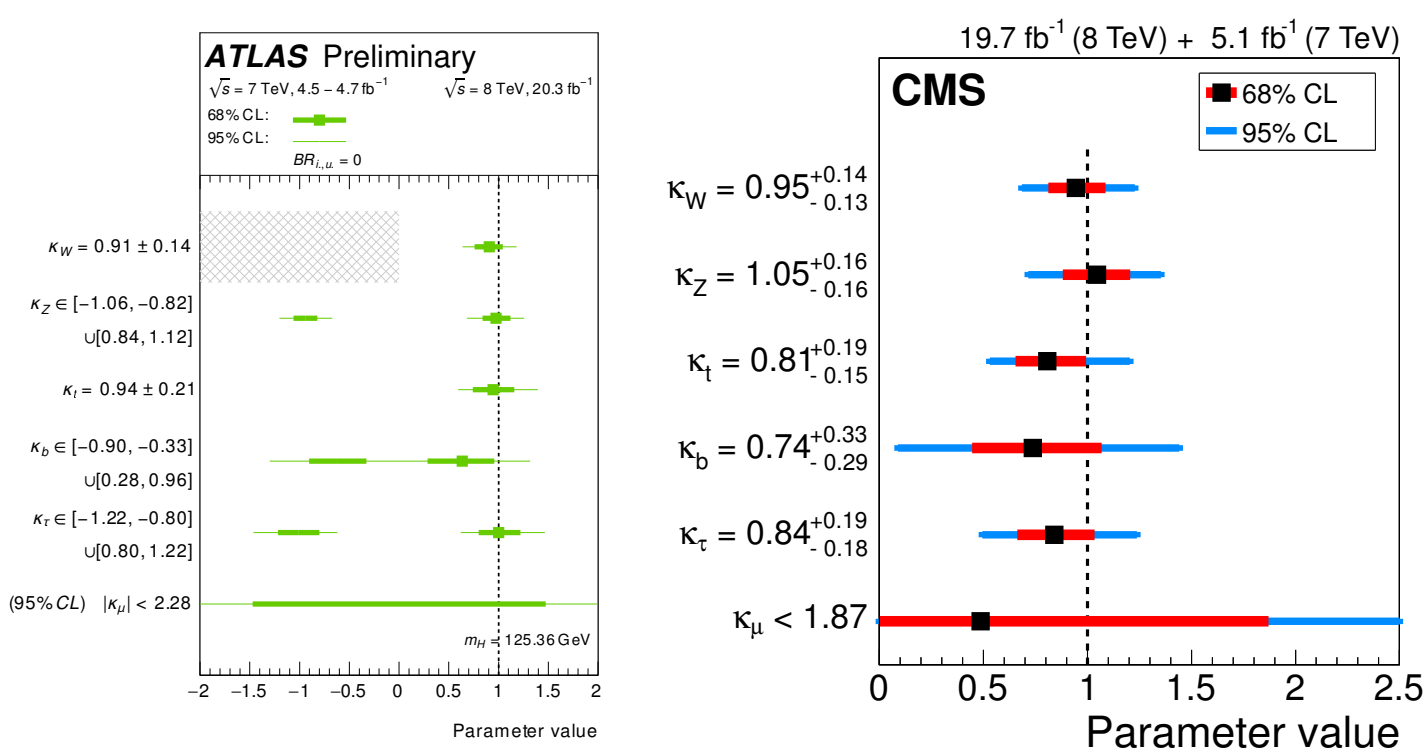

Figure 2: Best fit values of coupling scaling parameters $\kappa$ under the assumption that the Higgs boson does not interact with any particles other than those of the Standard Model. The CMS limit on $\kappa_{\mu}$ is given at $68 \%$ CL.

value $\kappa$ (and adding invisible decays) while keeping observed on-shell measurements the same, it is only possible to constrain ratios $\lambda_{i j}=\kappa_{i} / \kappa_{j}$ this way, as well as a single overall scale of SM interactions (denoted $\kappa_{g Z}$ ). In this model assumptions the loops are unresolved, i.e. there are additional $\kappa$ parameters for the $H \gamma \gamma, H g g$, and (for ATLAS) $H Z \gamma$ vertices. With this parametrization the ratios are determined to $\approx 14 \%$ for the best measured one ( $\lambda_{W Z}$, which probes custodial symmetry). New physics in the $H \gamma \gamma, H g g$, and $H t \bar{t}$ vertices is tested with $\lambda_{\gamma Z}$ and $\lambda_{t g}$. Results are shown in Fig. 3. The most notable discrepancy from the SM is the CMS determination of $\lambda_{t g}$, where the SM is not within the $95 \%$ CL band; however this is not seen by ATLAS, where agreement is seen just slightly beyond the $68 \%$ CL band.

It is possible to break the scaling degeneracy by introducing additional information or assumptions. For example, we can set $\mathrm{BR}_{i, u}=0$ (with a lot of model dependency). A slightly less blunt procedure is to introduce the constraints $\kappa_{V} \leq 1$ : in most models the couplings $\kappa_{W}$ and $\kappa_{Z}$ cannot exceed one (naively, this would mean the model provides "too much" electroweak symmetry breaking). Since it is not possible to scale all couplings with $\kappa<1$, this totally breaks the degeneracy, 

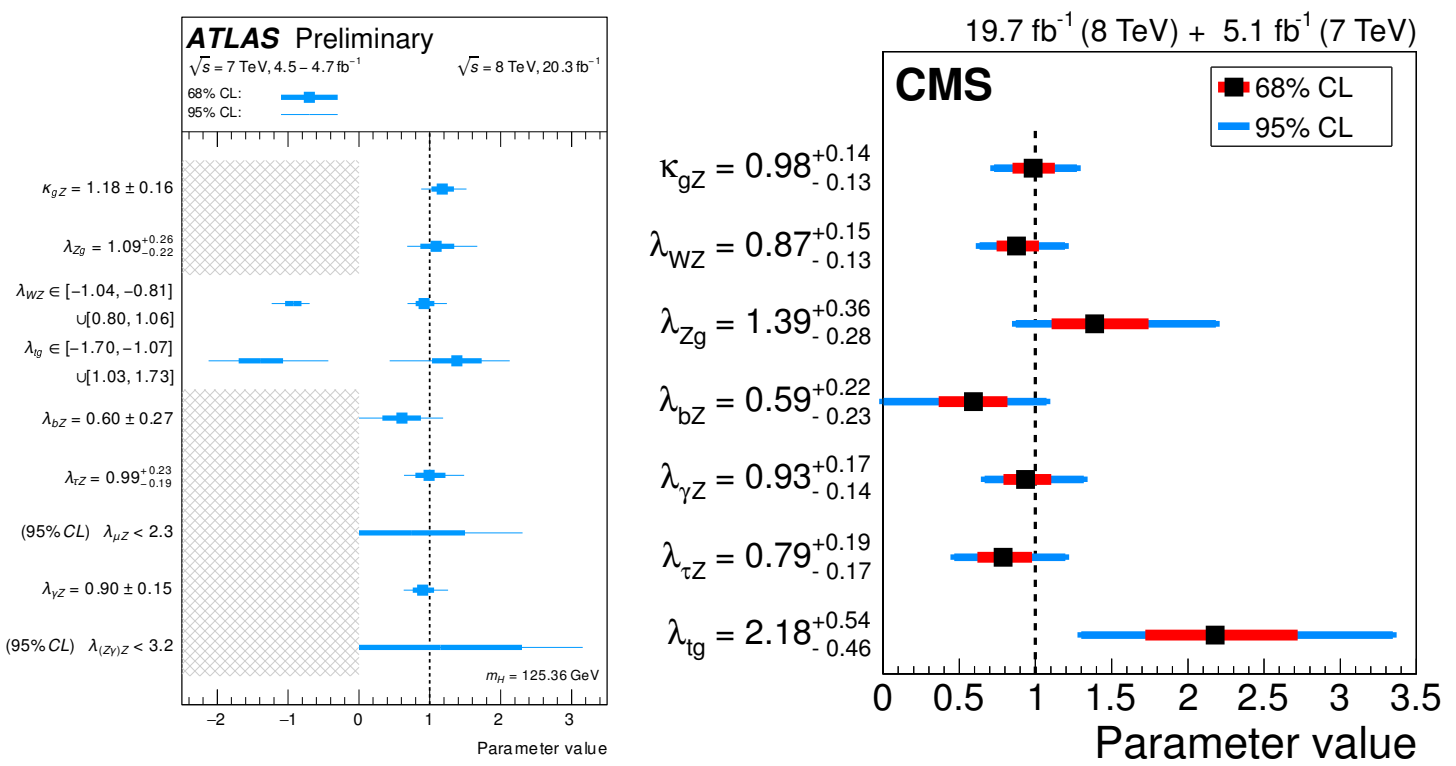

Figure 3: Constraints on ratios $\lambda_{i j}=\kappa_{i} / \kappa_{j}$ of Higgs boson coupling scaling factors for ATLAS (left) and CMS (right). This model is not sensitive to the overall Higgs width, but does allow BSM modifications to the effective couplings induced by loops.

at the cost of mild model-dependence. Finally, it is also possible to use the off-shell constraints, assuming energy-independence of the couplings; this also has the effect of constraining $\kappa_{V}$ from above with a somewhat milder cutoff.

Results are shown in Fig. 4. Depending on assumptions one can obtain indirect limits $\mathrm{BR}_{i, u} \lesssim$ $49-68 \%$ at $95 \%$ CL. Assuming that all additional Higgs boson decays are invisible (not undetected), CMS combines with direct searches for invisible Higgs boson decays (see Section 4) to obtain a limit $\mathrm{BR}_{u}<49 \%$, again at $95 \%$ CL.

\subsection{Spin/CP}

The Higgs boson is predicted to be a pure scalar $\left(J^{P}=0^{+}\right)$in the SM. BSM theories may produce bosons with different spin or $\mathrm{CP}$, or even states of mixed $\mathrm{CP}$. These can be probed at the LHC experiments.

The different spin/CP structures correspond to different allowed interactions in the Lagrangian. These can strongly alter many kinematic variables other than angular distributions. Compared to the $e^{+} e^{-}$environment, where angular analyses can typically exploit well-constrained initial states, the LHC Higgs boson spin/CP studies are best understood as limits on potential Lagrangian terms involving gauge bosons and a new resonance, which imply certain spin and CP properties of the new field. Using $H \rightarrow Z Z^{*} \rightarrow 4 \ell, H \rightarrow \gamma \gamma$, and $H \rightarrow W W^{*} \rightarrow \ell v \ell v$ decays, ATLAS and CMS have ruled out all tested non-SM (pure) Lagrangian terms at $>98 \% \mathrm{CL}$, disfavoring spin 1 and 2 hypotheses as well as $0^{-}$.

Recently ATLAS [9] and CMS [10] have probed the possibility of mixed terms: in addition to an SM-like term, having the Higgs boson couple via a CP-odd term (thus implying CP violation) 

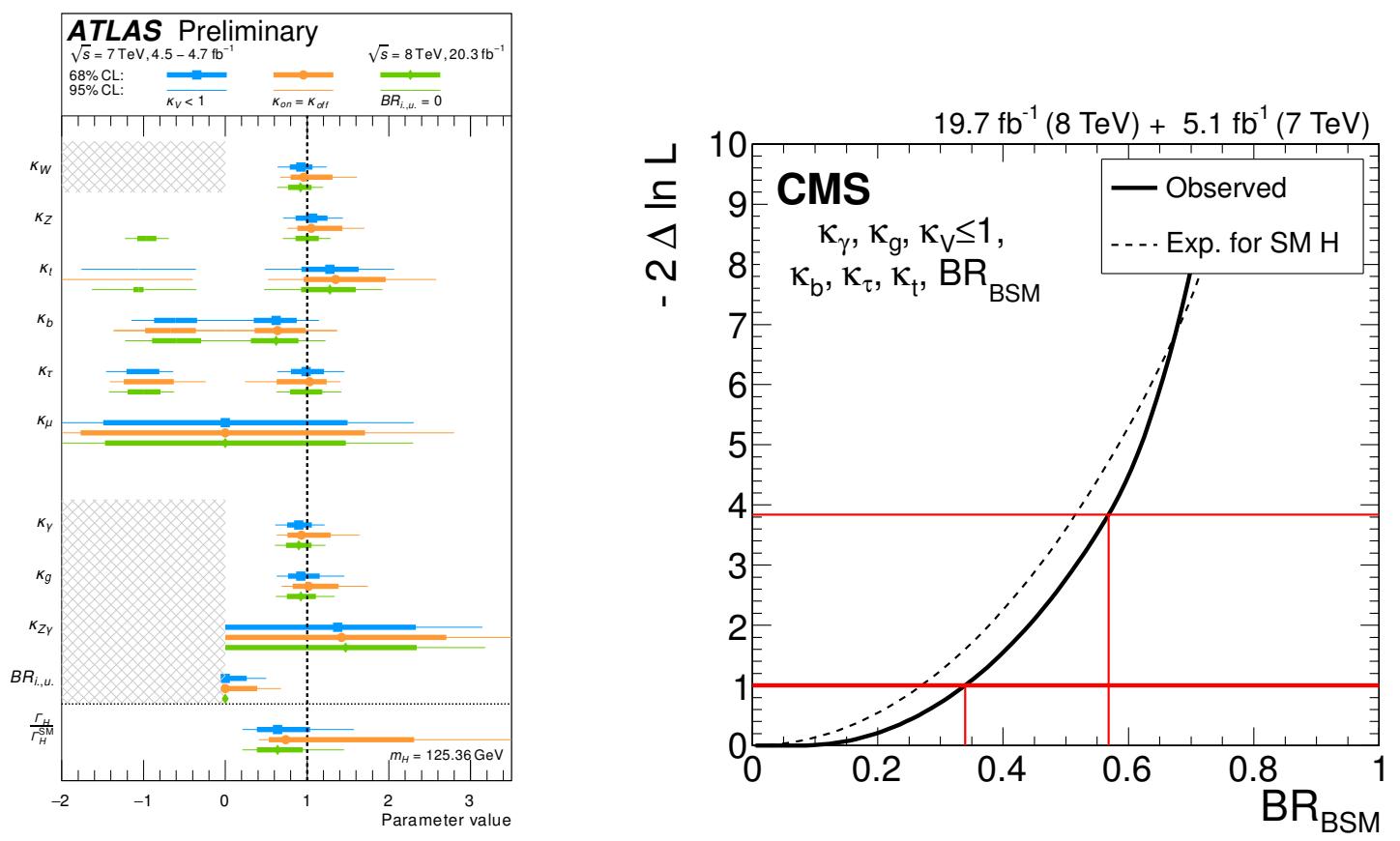

Figure 4: Left: ATLAS constraints on couplings allowing new contributions to loop diagrams $\left(\kappa_{\gamma}, \kappa_{g}\right.$, $\left.\kappa_{Z \gamma}\right)$ and invisible or undetected Higgs boson decays. Three hypotheses are considered: restricting $\kappa_{V} \leq 1$; using off-shell coupling limits and assuming on- and off-shell couplings are the same; and fixing $\mathrm{BR}_{i, u}=0$. Right: Likelihood function for $\mathrm{BR}_{i, u}$ (here denoted $\mathrm{BR}_{\mathrm{BSM}}$ ) as determined by CMS in a similar model. The constraint $\kappa_{V} \leq 1$ is imposed.

or through a higher-dimensional $0^{+}$interaction. The limits are primarily set through $H \rightarrow Z Z^{*}$ and $H \rightarrow W W^{*}$ decays; in particular the former provides a fully-reconstructed final state with a wealth of angles and masses sensitive to the dynamics of the decay. No evidence for a departure from the SM-like $0^{+}$behavior is seen.

\section{Non-SM Flavor Structure}

The Higgs boson of the Standard Model has flavor-diagonal interactions. However this is not necessarily the case in extensions of the SM; symmetries need to be imposed on generic two Higgs doublet models (2HDM) to avoid unacceptable flavor changing neutral currents (FCNC). However FCNC involving the third generation is not as well constrained from low energy experiments as that involving the first and second generations only, and (due to likely larger couplings) is precisely what can be probed with direct measurements of Higgs boson interactions.

\subsection{Quark FCNC}

Searches have been performed for the vertices $t H c$ and $t H u$. These would be seen in decays of top quarks. Over five million $t \bar{t}$ pairs per detector have been produced, which can be used to search for rare decays $t \rightarrow H q$. Searches have been performed using $H \rightarrow \gamma \gamma[11,12]$ and 
$H \rightarrow W W^{*} / Z Z^{*} / \tau \tau[12]$ decays and have set limits of

$$
\begin{aligned}
\mathscr{B}(t \rightarrow H q) & <0.79 \%(0.51 \% \text { expected }) \text { [ATLAS } \gamma \gamma] \\
& <0.56 \%(0.65 \% \text { expected }) \text { [CMS combined] }
\end{aligned}
$$

\subsection{Lepton Flavor Violation}

Turning to the lepton sector, decays $H \rightarrow \mu e$ are strongly constrained by the non-observation of $\mu \rightarrow e \gamma$. However the constraints from $\tau \rightarrow \mu \gamma$ and $\tau \rightarrow e \gamma$ are much weaker, leaving open the possibility for lepton flavor violating decays $H \rightarrow \tau \mu$ and $H \rightarrow \tau e$ to have branching fractions up to $\approx 10 \%$.

CMS has performed a search [13] for the decay $H \rightarrow \tau \mu$, using both $\tau \rightarrow e v \bar{v}$ and $\tau \rightarrow$ hadrons. The events are split into categories based on the $\tau$ lepton decay mode and the number of jets in the event; this provides signal regions with different background composition and populated by different Higgs production mechanisms. The $\tau \mu$ collinear mass ${ }^{1}$ is used as a discriminant. The 95\% CL upper limit on $\mathscr{B}(H \rightarrow \tau \mu)$ is found to be $1.51 \%$ ( $0.75 \%$ expected). The best fit branching fraction is $0.84_{-0.37}^{+0.39}$, corresponding to a $2.4 \sigma$ excess. The mass distribution is shown in Fig. 5 .
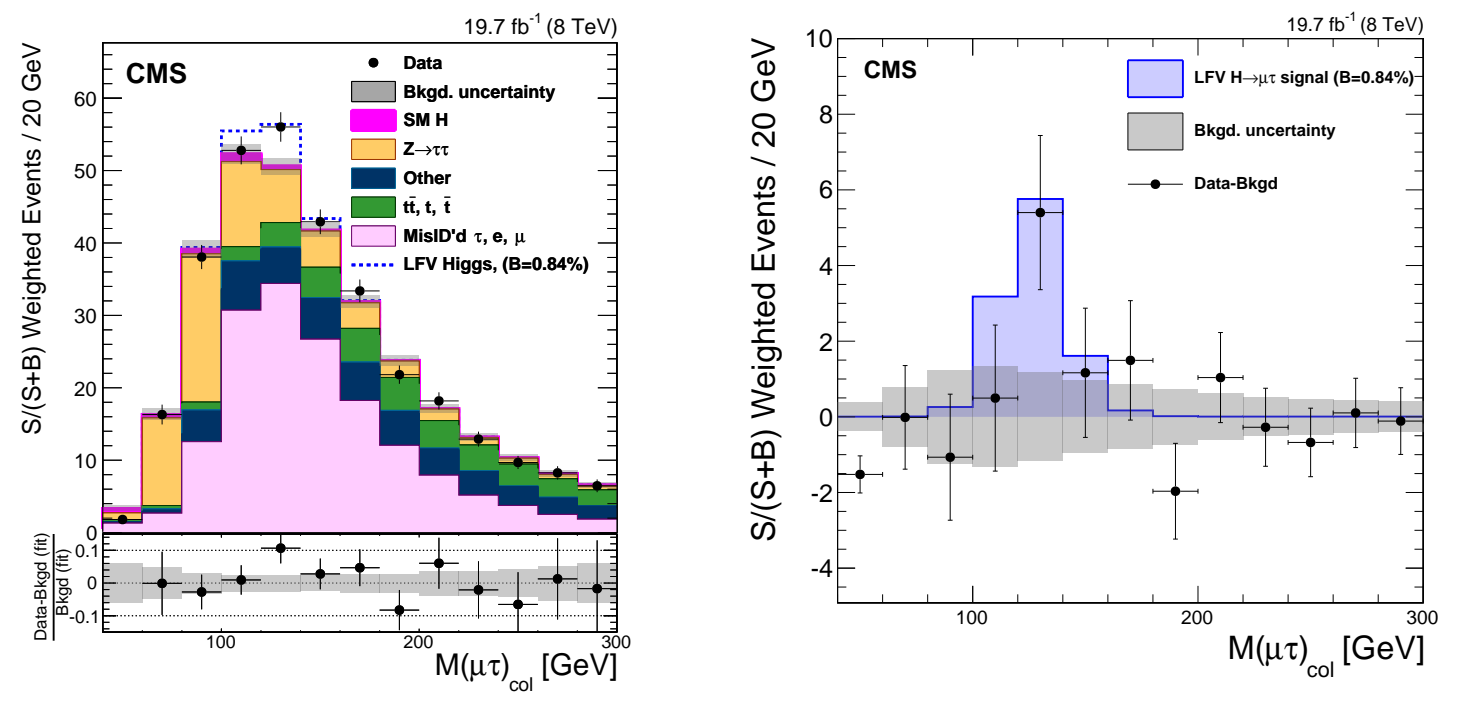

Figure 5: Results from CMS $H \rightarrow \tau \mu$ search. Left: $\tau \mu$ collinear mass in all categories, with each category weighted by $S /(S+B)$. Right: background-subtracted version of the distribution on the left.

\section{Invisible Higgs Boson Decays}

As discussed in Section 2, there is significant room for invisible or undetected Higgs boson decays. It is plausible that the Higgs boson has interactions with additional particles in a dark

\footnotetext{
${ }^{1}$ In the collinear approximation, the four-vectors of the decaying $\tau$ leptons are reconstructed by assuming that the neutrinos are collinear with the visible tau decay products and that the missing transverse momentum $E_{\mathrm{T}}^{\text {miss }}$ of the event arises purely from these neutrinos. The collinear mass is the di- $\tau$ mass obtained from these four-vectors.
} 
sector, because the operator $H^{\dagger} H$ is a dimension two gauge invariant scalar and can be combined easily with additional SM singlets.

Dedicated searches can be made for invisible decays of the Higgs boson. These searches use vector boson fusion $(p p \rightarrow q q H)[14,15]$ or vector boson associated production $(p p \rightarrow V H)$ [1618] of the Higgs boson. If the Higgs boson has large $p_{\mathrm{T}}$, a decay to invisible particles will result in large observed missing transverse momentum $E_{\mathrm{T}}^{\text {miss }}$. The additional particles (the pair of forward jets in the case of vector boson fusion, or the vector boson in the case of associated production) serve as a tag to enhance the signal-to-background ratio. For vector boson associated production, both $Z \rightarrow \ell \ell$ and $W / Z \rightarrow q \bar{q}$, in particular $Z \rightarrow b \bar{b}$, decays are exploited.

The most powerful searches are in vector boson fusion decays, where the following limits are obtained:

$$
\begin{aligned}
\mathrm{BR}_{u} & <29 \%(35 \% \text { expected) [ATLAS [14]] } \\
& <57 \% \text { (40\% expected) [CMS [15]] }
\end{aligned}
$$

CMS combines the vector boson fusion and $Z H$ production analyses to obtain the limit $\mathrm{BR}_{u}<$ $47 \%$ (35\% expected) [15]. It should be noted that these limits assume the SM production cross section for all processes. These are somewhat more powerful limits than the indirect ones from the coupling fits, although those do not assume SM production rates.

Assuming that the Higgs boson couples to a pair of dark matter (DM) particles $\chi \chi$, the Higgs boson can serve as a $t$-channel mediator of DM-nucleon scattering $(p, n) \chi \rightarrow(p, n) \chi$. The cross section depends on a Higgs boson-nucleon coupling form factor which has been calculated on the lattice. The non-observation of invisible Higgs boson decays can be translated into a limit on the spin-independent DM-nucleon scattering cross section for $m_{\chi}<m_{H} / 2$, assuming the DM-nucleon interaction is mediated only by Higgs boson exchange. These reinterpretations are shown in Fig. 6. They are competitive with direct detection experiments and are the strongest limits available for $m_{\chi} \lesssim 7 \mathrm{GeV}$ in this "Higgs portal" scenario.

\section{Searches for New States}

Extended electroweak symmetry breaking sectors will typically include other particles. Two Higgs doublet models (2HDM) will contain two scalars $h$ and $H$, charged scalars $H^{ \pm}$, and a pseudoscalar $A$. The charged scalars $H^{ \pm}$will have decays to fermions similar to those of the $W^{ \pm}$. Depending on masses, heavier Higgs sector particles may decay to lighter ones (e.g. $H \rightarrow h h$ ).

\subsection{Searches for $H^{+}$}

The parameters of the charged scalars $\mathrm{H}^{+}$can be constrained strongly by lower-energy flavor physics probes. As they mediate interactions similar in structure to those of the $W^{ \pm}$(although strongly dependent on the mass of the fermions involved), they modify the rate for electroweak processes such as $b \rightarrow s \gamma, B^{+} / D^{+} \rightarrow \tau \nu$, and $B \rightarrow D^{(*)} \tau \nu$. Indeed initial BaBar measurements of $B \rightarrow D^{(*)} \tau \nu$ [19] suggested that no 2HDM of type II (for example, the Higgs sector of minimal supersymmetry) was compatible with data, although results presented at this conference have weakened this conclusion $[20,21]$. 

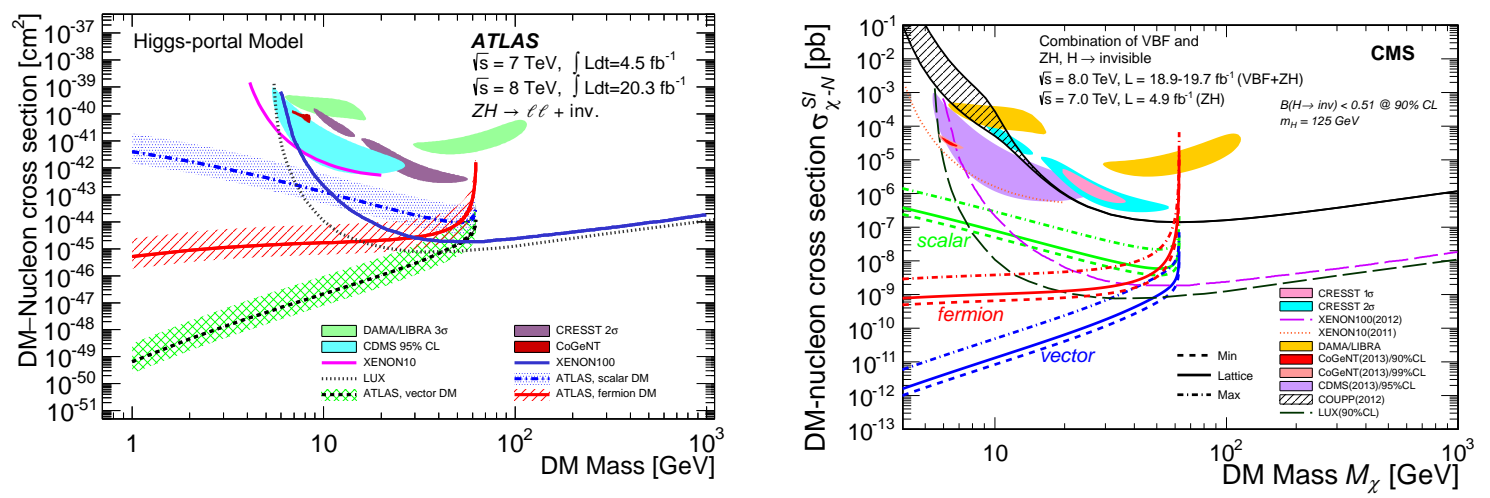

Figure 6: Limits on dark matter-nucleon cross sections derived from Higgs boson invisible decay searches, interpreted in the context of Higgs portal models. The ATLAS result is taken from Ref. [17]. The CMS result is taken from Ref. [18] and does not include the latest vector boson fusion limit [15].

Ignoring higher order corrections and possible decays of the $\mathrm{H}^{+}$to other states in the Higgs sector, the production and decay of the $H^{+}$can be understood in terms of the parameters $m_{H^{+}}$and $\tan \beta$, the ratio of vacuum expectation values of the two doublets.

Because of the dependence of the couplings on the mass of the final state fermions, the most significant production (and decay) vertices are $H^{ \pm} t b$ and $H^{ \pm} \tau \nu$ ( $H^{ \pm} c s$ can be relevant to searches in some regions of parameter space). Search strategies can be divided into the low and high $m_{H^{+}}$ regions:

- $m_{H^{+}}<m_{t}$ : in this case the process $t \rightarrow H^{+} b$ can occur, and searches target $t \bar{t}$ production with subsequent decay of one of the top quarks to $H^{+}$. Since $H^{+}$decays to top quarks are not kinematically allowed in this region, the dominant decay is $H^{+} \rightarrow \tau \nu$.

- $m_{H^{+}}>m_{t}$ : in this case the $H^{+}$is mostly produced in association with a single top quark (analogously to $W t$ production). The $H^{+}$will then typically decay to $t \bar{b}$, although $\tau v$ can still have an appreciable branching fraction and contribute to the sensitivity.

In the Type-II $2 \mathrm{HDM}$, the $H t b$ coupling is minimized for $\tan \beta \approx 8$, which will minimize both the $t \rightarrow H^{+} b$ branching fraction (for light $H^{+}$) and the $t b H^{+}$production cross section. The $t b H^{+}$ cross section varies over three orders of magnitude as $\tan \beta$ varies from 0.1 to 60 (see Fig. 7). Direct search limits from the LHC, interpreted in the $m_{H^{+}}$vs. $\tan \beta$ plane, will therefore always be strongest at very low and very high $\tan \beta$.

In searches using $H^{+} \rightarrow \tau \nu$, if there are no additional neutrinos in the event it is possible to reconstruct a transverse mass variable $m_{\mathrm{T}}$ from the $\tau$ candidate and the $E_{\mathrm{T}}^{\text {miss }}$ with a Jacobian edge at $m_{H^{+}}$. Therefore the most typical $H^{+} \rightarrow \tau \nu$ searches target all-hadronic decays of the additional top quark in the event. Both ATLAS [22] and CMS [23] have performed this search in low and high mass regions and achieve similar sensitivity to each other. The results are shown in Fig. 8. Almost all values of $m_{H^{+}}$and $\tan \beta$ are excluded for $m_{H^{+}}<m_{t}$, with only a small region open for 140-150 $\mathrm{GeV} \lesssim m_{H^{+}}<m_{t}$ and $\tan \beta \approx 5-15$. At high $m_{H^{+}}$the situation is more open, with a small amount 
of parameter space at high $\tan \beta$ and $m_{H^{+}}$just above the top mass excluded. A CMS analysis using leptonic top decays is also available with significantly weaker limits [24].

Searches with $H^{+} \rightarrow t \bar{b}$ are significantly harder to perform as reconstruction of the $H^{+}$is much more difficult. CMS has performed a search in this channel, using leptonic decays of both the top quark produced in association with the $H^{+}$and the top quark produced in its decay [24].
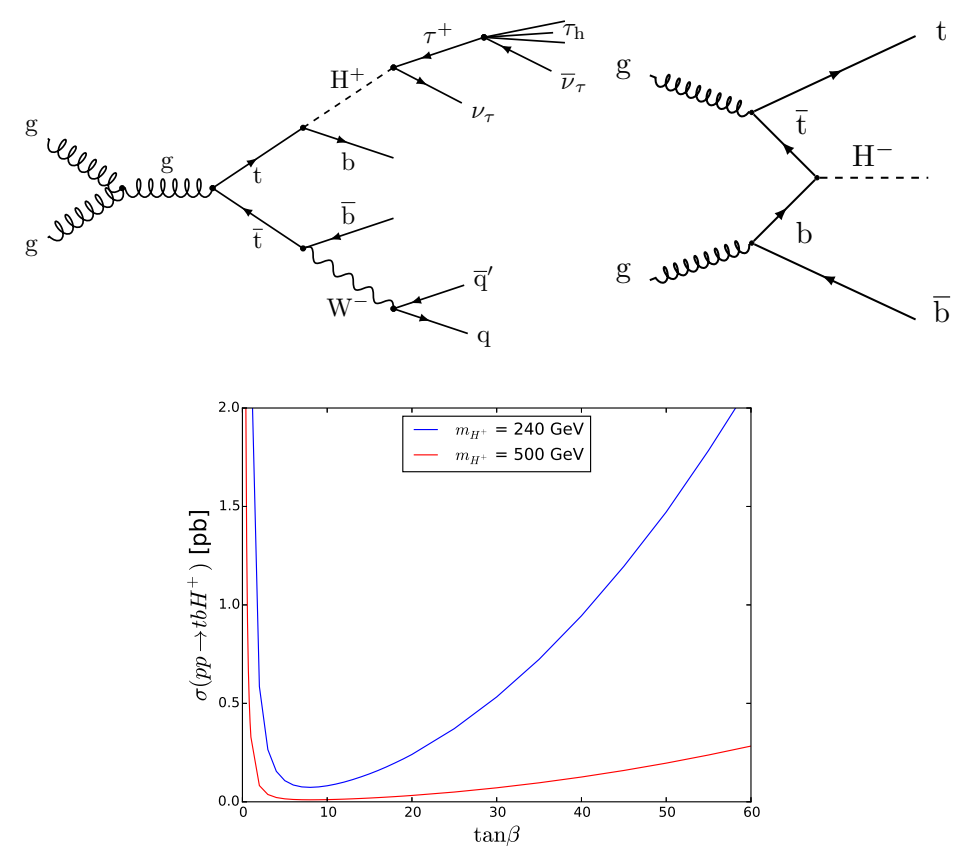

Figure 7: Top row: Feynman diagrams for production of $H^{+}$in $t \bar{t}$ decays (top left) and in association with $t \bar{b}$ (top right). Bottom: cross section for production of a heavy $H^{+}$for two choices of $m_{H^{+}}$in the Type-II $2 \mathrm{HDM}[6,25,26]$. Direct searches are least sensitive at the minimum of $\tan \beta \approx 8$. (Values taken from Ref. [27].)

In addition, there are searches focusing on $H^{+} \rightarrow c \bar{s}[29,30]$, a mode that can be relevant for light $H^{+}$. These focus on hadronic decays $t \rightarrow H^{+} b \rightarrow c \bar{s} b$. The non- $b$-tagged jets in this triplet will have invariant mass close to $m_{H^{+}}$, as opposed to normal top quark decays, in which the jets will have mass close to $m_{W^{+}}$. A fit to the dijet mass distribution is used to constrain any possible $H^{+}$contribution.

The decay $H^{+} \rightarrow W^{+} Z$ is not allowed in 2HDM; however, in more complex models (such as those involving Higgs triplets) it is allowed. A search has been performed by ATLAS in vector boson fusion production, decaying to $W\left(\rightarrow q \bar{q}^{\prime}\right) Z(\rightarrow \ell \ell)$ [31]. No evidence of a $W Z$ resonance is seen.

\subsection{Searches for Light Pseudoscalars}

The minimal supersymmetric standard model (MSSM) has some fine tuning concerns that can be alleviated by adding an additional singlet which also obtains a vacuum expectation value. This model is referred to as the next-to-minimal supersymmetric standard model (NMSSM) and further extends the Higgs sector of the MSSM; in particular an additional pseudoscalar $(a)$ is expected 

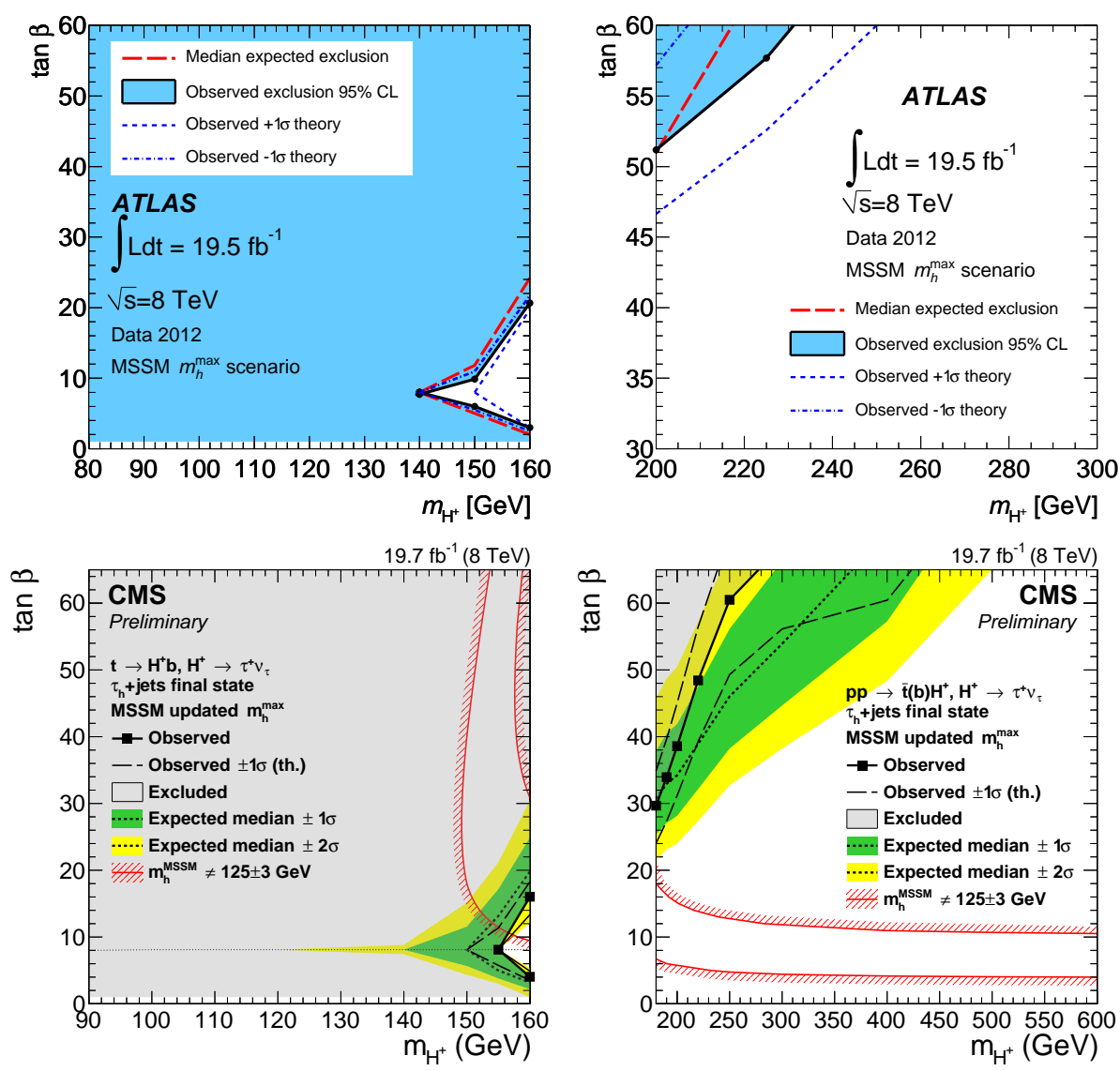

Figure 8: Exclusions in the $m_{H^{+}}-\tan \beta$ plane from $H^{+} \rightarrow \tau v$ searches at ATLAS [22] and CMS [23]. The exclusions are presented in the context of an MSSM scenario [28] which allows calculation of higher order corrections.

which may be very light $(\mathscr{O}(\mathrm{GeV}))$. This particle will decay to the accessible fermions with a coupling that depends on mass. This model motivates searches for light particles decaying to $\mu \mu$ and $\tau \tau$. An extensive suite of searches for such particles in quarkonium decays has been performed by the $B$-factory experiments. CMS has also searched for singly produced $a$ in gluon gluon fusion [32] and for decays $H \rightarrow a a \rightarrow 4 \mu$ [33].

ATLAS has recently performed a search for the decay $H \rightarrow a a \rightarrow \mu \mu \tau \tau$ [34], a channel previously investigated by the $\mathrm{D} \varnothing$ collaboration [35]. This is more sensitive than $a a \rightarrow 4 \mu$ for the region $m_{a}>2 m_{\tau}$. This search is sensitive to any heavy resonance $H$ that decays to a pair of light pseudoscalars, although an obvious candidate for such a resonance is the $125 \mathrm{GeV}$ Higgs boson. The final state is $\mu \mu+(e, \mu) \tau_{\text {had }}$; the hadronically-decaying tau candidate is reconstructed using a simple track selection. Because the two $a$ arise in the decay of a heavy object, they are expected to be boosted and to be back to back; angular separation and $p_{\mathrm{T}}(a)$ requirements are imposed to suppress background. The dimuon mass spectrum is scanned for evidence of a dimuon resonance; none is seen. 


\subsection{Searches for di-Higgs boson Production}

Searches for di-Higgs boson production have been performed by ATLAS and CMS. This production may be resonant or non-resonant. Resonant production would, for example, be expected in the 2HDM, with the heavy $C P$-even scalar decaying to two $125 \mathrm{GeV}$ Higgs bosons. Non-resonant production has an extremely small cross section in the SM and is not expected to be observed before the end of the HL-LHC program. Observation of di-Higgs boson production of any kind is therefore a marker of beyond-SM physics.

The searches are characterized by the Higgs boson decays used. The channels targeted so far are $4 b$ [36, 37], $b b \gamma \gamma$ [38, 39], and leptons and $\gamma \gamma$ [12]. At low $m(H H)$ the $b b \gamma \gamma$ channel is most sensitive, due to the high mass resolution of the diphoton decay. At high $m(H H)$ the $4 b$ channel takes over as the pairs of $b$-tagged jets become boosted and combinatorics are easier to resolve, so the higher branching fraction of $H \rightarrow b \bar{b}$ wins. No significant excess is seen in any of the searches.

\section{Conclusion}

Flavor physics seeks to illuminate high energy scales though precision measurement of low scale processes. These scales, in particular those of the electroweak symmetry breaking sector, are becoming available for direct study at the LHC. There is a strong interplay of of direct and indirect limits and, while some flavor physics limits are likely to remain stronger than any conceivable LHC constraints, others are already or will become dominated by direct searches. Certainly, in the event of observation of new physics in either direct or indirect searches, the other will be critical to properly characterize the new interactions.

\section{References}

[1] ATLAS Collaboration, The ATLAS Experiment at the CERN Large Hadron Collider, JINST 3 (2008) S08003.

[2] CMS Collaboration, The CMS experiment at the CERN LHC, JINST 3 (2008) S08004.

[3] ATLAS and CMS Collaborations, Combined Measurement of the Higgs Boson Mass in pp Collisions at $\sqrt{s}=7$ and 8 TeV with the ATLAS and CMS Experiments, Phys. Rev. Lett. 114 (2015) 191803, arXiv:1503.07589 [hep-ex] .

[4] ATLAS Collaboration, Measurements of the Higgs boson production and decay rates and coupling strengths using pp collision data at $\sqrt{s}=7$ and $8 \mathrm{TeV}$ in the ATLAS experiment, ATLAS-CONF-2015-007 (2015).

[5] CMS Collaboration, Precise determination of the mass of the Higgs boson and tests of compatibility of its couplings with the standard model predictions using proton collisions at 7 and 8 TeV, Eur. Phys. J. C 75 (2015) 212, arXiv:1412.8662 [hep-ex] .

[6] S. Heinemeyer et al., Handbook of LHC Higgs Cross Sections: 3. Higgs Properties, CERN-2013-004 (2013), arXiv:1307.1347 [hep-ph]. 
[7] ATLAS Collaboration, Constraints on the off-shell Higgs boson signal strength in the high-mass ZZ and WW final states with the ATLAS detector, Eur. Phys. J. C 75 (2015) 335, arXiv:1503.01060 [hep-ex].

[8] CMS Collaboration, Constraints on the Higgs boson width from off-shell production and decay to Z-boson pairs, Phys. Lett. B 736 (2014) 64, arXiv:1405.3455 [hep-ex] .

[9] ATLAS Collaboration, Study of the spin and parity of the Higgs boson in diboson decays with the ATLAS detector, CERN-PH-EP-2015-114 (2015), arXiv:1506.05669 [hep-ex].

[10] CMS Collaboration, Constraints on the spin-parity and anomalous HVV couplings of the Higgs boson in proton collisions at 7 and 8 TeV, Phys. Rev. D 92 (2015) 012004, arXiv:1411.3441 [hep-ex].

[11] ATLAS Collaboration, Search for top quark decays $t \rightarrow q H$ with $H \rightarrow \gamma \gamma$ using the ATLAS detector, JHEP 1406 (2014) 008, arXiv: 1403.6293 [hep-ex] .

[12] CMS Collaboration, Searches for heavy Higgs bosons in two-Higgs-doublet models and for $t \rightarrow$ ch decay using multilepton and diphoton final states in pp collisions at $8 \mathrm{TeV}$, Phys. Rev. D 90 (2014) 112013, arXiv:1410.2751 [hep-ex] .

[13] CMS Collaboration, Search for lepton-flavour-violating decays of the Higgs boson, CERN-PH-EP-2015-027 (2015), arXiv:1502.07400 [hep-ex] .

[14] ATLAS Collaboration, Search for an Invisibly Decaying Higgs Boson Produced via Vector Boson Fusion in pp Collisions at $\sqrt{s}=8 \mathrm{TeV}$ using the ATLAS Detector at the LHC, ATLAS-CONF-2015-004 (2015).

[15] CMS Collaboration, Search for invisible decays of Higgs bosons in the vector boson fusion production mode, CMS-PAS-HIG-14-038 (2015).

[16] ATLAS Collaboration, Search for invisible decays of the Higgs boson produced in association with a hadronically decaying vector boson in pp collisions at $\sqrt{s}=8 \mathrm{TeV}$ with the ATLAS detector, Eur. Phys. J. C 75 (2015) 337, arXiv:1504.04324 [hep-ex] .

[17] ATLAS Collaboration, Search for Invisible Decays of a Higgs Boson Produced in Association with a Z Boson in ATLAS, Phys. Rev. Lett 112 (2014) 201802, arXiv:1402.3244 [hep-ex].

[18] CMS Collaboration, Search for invisible decays of Higgs bosons in the vector boson fusion and associated ZH production modes, Eur. Phys. J. C 74 (2014) 2980, arXiv: 1404.1344 [hep-ex].

[19] BaBar Collaboration, J. Lees et al., Evidence for an excess of $\bar{B} \rightarrow D^{(*)} \tau^{-} \bar{v}_{\tau}$ decays, Phys. Rev. Lett 109 (2012) 101802, arXiv:1205.5442 [hep-ex]. 
[20] Belle Collaboration, M. Huschle et al., Measurement of the branching ratio of $\bar{B} \rightarrow D^{(*)} \tau^{-} \bar{v}_{\tau}$ relative to $\bar{B} \rightarrow D^{(*)} \ell^{-} \bar{v}_{\ell}$ decays with hadronic tagging at Belle, arXiv: 1507.03233 [hep-ex].

[21] LHCb Collaboration, R. Aaij et al., Measurement of the ratio of branching fractions $\left.\mathscr{B}\left(\bar{B}^{0} \rightarrow D^{*+} \tau^{-} \bar{v}_{\tau}\right)\right) / \mathscr{B}\left(\bar{B}^{0} \rightarrow D^{*+} \mu^{-} \bar{v}_{\mu}\right)$, CERN-PH-EP-2015-150 (2015), arXiv:1506.08614 [hep-ex].

[22] ATLAS Collaboration, Search for charged Higgs bosons decaying via $H^{ \pm} \rightarrow \tau^{ \pm} v$ in fully hadronic final states using pp collision data at $\sqrt{s}=8 \mathrm{TeV}$ with the ATLAS detector, JHEP 1503 (2015) 088, arXiv:1412.6663 [hep-ex] .

[23] CMS Collaboration, Search for charged Higgs bosons with the $H^{+} \rightarrow \tau v$ decay channel in the fully hadronic final state at $\sqrt{s}=8 \mathrm{TeV}$, CMS-PAS-HIG-14-020 (2014).

[24] CMS Collaboration, Search for a heavy charged Higgs boson in proton-proton collisions at $\sqrt{s}=8 \mathrm{TeV}$ with the CMS detector, CMS-PAS-HIG-13-026 (2014).

[25] M. Flechl, R. Klees, M. Kramer, M. Spira, and M. Ubiali, Improved cross-section predictions for heavy charged Higgs boson production at the LHC, Phys. Rev. D 91 (2015) 075015, arXiv:1409.5615 [hep-ph].

[26] E. L. Berger, T. Han, J. Jiang, and T. Plehn, Associated production of a top quark and a charged Higgs boson, Phys. Rev. D 71 (2005) 115012, arXiv: hep-ph/0312286 [hep-ph] .

[27] https://twiki.cern.ch/twiki/bin/view/LHCPhysics/LHCHXSWGMSSMCharged.

[28] M. Carena, S. Heinemeyer, O. Stål, C. Wagner, and G. Weiglein, MSSM Higgs Boson Searches at the LHC: Benchmark Scenarios after the Discovery of a Higgs-like Particle, Eur. Phys. J. C 73 (2013) 2552, arXiv:1302.7033 [hep-ph] .

[29] ATLAS Collaboration, Search for a light charged Higgs boson in the decay channel $H^{+} \rightarrow c \bar{s}$ in $t \bar{t}$ events using pp collisions at $\sqrt{s}=7 \mathrm{TeV}$ with the ATLAS detector, Eur. Phys. J. C 73 (2013) 2465, arXiv:1302.3694 [hep-ex] .

[30] CMS Collaboration, Search for $H^{+} \rightarrow c \bar{s}$ decay, CMS-PAS-HIG-13-035 (2014).

[31] ATLAS Collaboration, Search for a Charged Higgs Boson Produced in the Vector-Boson Fusion Mode with Decay $H^{ \pm} \rightarrow W^{ \pm} Z$ using pp Collisions at $\sqrt{s}=8 \mathrm{TeV}$ with the ATLAS Experiment, Phys. Rev. Lett 114 (2015) 231801, arXiv:1503.04233 [hep-ex] .

[32] CMS Collaboration, Search for a light pseudoscalar Higgs boson in the dimuon decay channel in pp collisions at $\sqrt{s}=7 \mathrm{TeV}$, Phys. Rev. Lett 109 (2012) 121801, arXiv:1206.6326 [hep-ex].

[33] CMS Collaboration, Search for a non-standard-model Higgs boson decaying to a pair of new light bosons in four-muon final states, Phys. Lett. B 726 (2013) 564-586, arXiv:1210.7619 [hep-ex]. 
[34] ATLAS Collaboration, Search for Higgs bosons decaying to aa in the $\mu \mu \tau \tau$ final state in $p p$ collisions at $\sqrt{s}=8 \mathrm{TeV}$ with the ATLAS experiment, CERN-PH-EP-2015-057 (2015), arXiv:1505.01609 [hep-ex].

[35] DØ Collaboration, V. Abazov et al., Search for NMSSM Higgs bosons in the $h \rightarrow a a \rightarrow \mu \mu \mu \mu, \mu \mu \tau \tau$ channels using p p collisions at $\sqrt{s}=1.96 \mathrm{TeV}$, Phys. Rev. Lett 103 (2009) 061801, arXiv:0905.3381 [hep-ex].

[36] ATLAS Collaboration, Search for Higgs boson pair production in the $b \bar{b} b \bar{b}$ final state from pp collisions at $\sqrt{s}=8 \mathrm{TeV}$ with the ATLAS detector, CERN-PH-EP-2015-099 (2015), arXiv:1506.00285 [hep-ex].

[37] CMS Collaboration, Search for di-Higgs resonances decaying to 4 bottom quarks, CMS-PAS-HIG-14-013 (2014).

[38] ATLAS Collaboration, Search For Higgs Boson Pair Production in the $\gamma \gamma b \bar{b}$ Final State using pp Collision Data at $\sqrt{s}=8 \mathrm{TeV}$ from the ATLAS Detector, Phys. Rev. Lett 114 (2015) 081802, arXiv:1406.5053 [hep-ex] .

[39] CMS Collaboration, Search for the resonant production of two Higgs bosons in the final state with two photons and two bottom quarks, CMS-PAS-HIG-13-032 (2014). 